\title{
LA INNOVACIÓN EN EL SECTOR TURÍSTICO: EL CASO DE BALEARES
}

\author{
Marta Jacob \\ Eugeni Aguiló2
}

Universitat de les Illes Balears

\begin{abstract}
Resumen:
Este trabajo se inicia con la descripción del marco teórico para el análisis y la medición de la innovación en sectores de servicios en general y en turismo en particular. Se realiza un análisis de la evidencia empírica disponible sobre la actividad innovadora del sector turístico balear y su capacidad de transferir tecnologías y conocimientos a otros destinos menos desarrollados, centrándonos en los resultados que arrojan dos trabajos (Jacob et al., 2004; Jacob y Groizard, 2007). Básicamente, la innovación es un fenómeno muy frecuente en el sector turístico balear, la innovación es principalmente tecnológica y del área de tecnologías de la información y comunicación (TIC) y del área medioambiental. Asimismo, las actividades de innovación de las cadenas hoteleras multinacionales (como las de origen balear) en países de renta media y baja tienen un enorme potencial para promocionar el desarrollo doméstico.
\end{abstract}

Palabras clave: innovación, transferencia de tecnología, sector turístico, Islas Baleares

Resumo: A innovación no sector turístico: o caso das Baleares.

Este traballo iníciase cunha descrición do marco teórico empregado para a análise e a medida da innovación en sectores de servizos en xeral e no turismo en particular. Realízase unha análise da evidencia empírica dispoñible sobre a actividade innovadora do sector turístico balear e a súa capacidade de transferir tecnoloxías e coñecementos a outros destinos menos desenvolvidos, na cal nos centramos nos resultados de dous traballos (Jacob et al., 2004; Jacob e Groizard, 2007). Basicamente, a innovación é un fenómeno moi frecuente no sector turístico balear. Falamos dunha innovación principalmente tecnolóxica, da área de tecnoloxías da información e comunicación (TIC) e da área ambiental. Así mesmo, as actividades de innovación das cadeas hoteleiras multinacionais (como as de orixe balear) en países de renda media e baixa teñen un enorme potencial para fomentar o desenvolvemento doméstico.

Palabras clave: innovación, transferencia de tecnoloxía, sector turístico, Illas Baleares.

\footnotetext{
1 Marta Jacob. Departament d'Economia Aplicada, Universitat de les Illes Balears, Carretera de Valldemossa, Km. 7.5. 07122 Palma de Mallorca, Spain, e-mail: marta.jacob@uib.es Tel: +34-971-171320 Fax: +34-971172389

2 Eugeni Aguiló. Departament d'Economia Aplicada, Universitat de les Illes Balears, Carretera de Valldemossa, Km. 7.5.07122 Palma de Mallorca, Spain, e-mail: eugeniaguilo@uib.es Tel: +34-971-173134 Fax: +34-971-172389
} 


\begin{abstract}
Innovation in the tourism industry: The case of the Balearic Islands.
This work begins with a description of the theoretical framework used for the analysis and measurement of innovation in the services sectors in general, and tourism in particular. The available empirical evidence on the innovative activity of the Balearic Islands' tourism industry and its capacity to transfer technologies and knowledge to other, less developed destinations is analysed, focusing on the results from two studies (Jacob et al., 2004; Jacob and Groizard, 2007). Basically, innovation is a very frequent phenomenon in the Balearic Islands' tourism industry, and it is primarily technological, in the areas of information and communications technologies (ICT) and the environment. Moreover, innovation activities by multinational hotel chains (and those based in the Balearic Islands) in medium- and low-income countries have a great potential to promote domestic development.
\end{abstract}

Key words: innovation, technology transfer, tourism industry, Balearic Islands.

\title{
1. INTRODUCCIÓN
}

La innovación es una herramienta clave para la competitividad de las empresas en todos los sectores económicos de forma que un sector económico competitivo será aquel que está formado por empresas que se adaptan a su entorno cambiante introduciendo novedades o mejoras en sus productos, procesos o en sus organizaciones. En las empresas de servicios la innovación se refiere a nuevos servicios o nuevas formas de producir o proveer los servicios, así como cambios significativos en productos ya existentes y en sus procesos de producción y distribución

La literatura sobre innovación en servicios es muy reciente y, de hecho, la evidencia empírica y estudios sobre innovación en servicios se centraban en sectores intensivos en conocimiento y de alta intensidad tecnológica. Sin embargo, el sector turístico es un sector que continuamente genera un mayor porcentaje de valor añadido a la economía mundial, y el principal sector económico para muchas regiones mediterráneas europeas como las Islas Baleares.

El sector turístico balear, y especialmente la industria hotelera, ha sido reconocido mundialmente por una competitividad mantenida durante décadas (e.g. Aguiló et al., 2005). Esa competitividad sólo ha sido posible gracias a la intensa actividad innovadora del sector.

El presente trabajo analiza por un lado, la reciente evidencia empírica sobre la actividad innovadora del sector turístico balear, uno de los mayores destinos turísticos europeos, y por otro lado, la intensidad y el tipo de tecnologías que las compañías multinacionales, como las empresas hoteleras de origen balear, transfieren a sus subsidiarias localizadas en países menos desarrollados.

El artículo se estructura en cuatro secciones: comienza con una breve revisión de la literatura sobre innovación en servicios, centrándonos en una tipología de innovación en servicios. La segunda sección describe la metodología utilizada para analizar y medir la actividad innovadora en empresas turísticas. La tercera sección analiza los principales resultados de la evidencia empírica sobre actividad innovadora en el sector turístico balear y sobre su transferencia de tecnología a otros destinos turísticos del Caribe y Latino-América. La última sección discute las conclusiones e implicaciones. 


\section{LA INNOVACIÓN EN SERVICIOS: ASPECTOS CONCEPTUALES Y TIPOLOGÍA}

El estudio de la innovación en sectores de servicios como el sector turístico es complejo debido a que la teoría y metodologías de innovación han sido principalmente desarrolladas para el análisis de la innovación tecnológica en el sector manufacturero y por tanto, no tienen en cuenta las peculiaridades del sector servicios. Sirilli y Evangelista (1998) resumen las principales características de los servicios que tienen implicaciones directas a la hora de definir y analizar la innovación en servicios:

- Relación estrecha entre producción y consumo o co-terminalidad de la producción y consumo de servicios.

- Intangibilidad y contenido intensivo en información de los productos y procesos en servicios.

- Papel fundamental de los recursos humanos como factor básico de competitividad.

- Importancia de los factores organizativos para la performance de la empresa.

En primer lugar, la relación estrecha entre la producción y el consumo dificulta la distinción entre innovación de producto y de proceso en servicios y lleva a una mayor orientación de las actividades de servicios hacia la adaptación-customización de los servicios a las necesidades de los clientes ${ }^{3}$. En segundo lugar, el alto contenido en información confiere a las Tecnologías de información y comunicación (TIC) un papel central en las actividades de innovación en las empresas de servicios y dificulta su almacenamiento y transporte (Torres y Jacob, 2001), así como la protección de innovaciones con métodos tradicionales como las patentes. En tercer lugar, el papel fundamental del factor humano en la organización y provisión de los servicios requiere una importante inversión en recursos humanos. Hipp et al. (2000) señalan que los conocimientos y las habilidades de la gente implicada en la producción y en las actividades de innovación son fundamentales para el desarrollo de estas actividades. Por último, como indican Sirilli y Evangelista (1998), la importancia de los factores organizativos en el sector de servicios requiere una revisión del concepto tradicional de innovación para poder tener en cuenta los cambios organizacionales.

Aparte de estas peculiaridades en servicios, que recomiendan una modificación de los conceptos y definiciones de innovación desarrollados inicialmente para el sector manufacturero para adaptarlos al sector servicios (e.g. Evangelista y Sirilli, 1995; Eurostat, 1995), la evidencia empírica sobre actividad de innovación en servicios sugiere por un lado, que las innovaciones no tecnológicas son muy comunes en servicios (por ejemplo, Gallouj, 1998; Sundbo y Gallouj, 1998; Miles, 1994) y por otro, que las innovaciones en servicios suelen ser más incrementales que radicales, consistentes en pequeños cambios incrementales en los procesos y procedimientos de forma que no requieren mucha I+D previa.

\footnotetext{
3 Tendencia que se da especialmente en el sector turístico en los últimos 10 años.
} 
Teniendo en cuenta estas características del sector servicios y la importancia de las innovaciones organizacionales, Sundbo y Gallouj (1998) identifican cuatro tipos de innovaciones en servicios:

- Innovaciones de producto: Consisten en la presentación de productos nuevos o mejorados significativamente al cliente.

- Innovaciones de proceso: Novedades o mejoras en los procesos de producción y de distribución y entrega de servicios. Estas innovaciones pueden ser de dos tipos: innovaciones en los procesos de producción e innovaciones en los procesos de distribución y entrega (provisión) de los servicios.

- Innovaciones de organización: Nuevas formas de organización de la empresa o de la gestión de la misma.

- Innovaciones de mercado: Nuevos comportamientos en el mercado, como encontrar un nuevo segmento de mercado o la introducción de la empresa en una nueva industria y en su mercado.

Es importante insistir en el hecho de que las innovaciones de organización contribuyen a la mejora de la productividad y la calidad de los servicios. Los cambios organizativos pueden estimular innovaciones para enfrentarse a nuevos desafíos y dificultades. Cabe también hacer la distinción entre innovaciones internas y externas de organización. Las primeras se producen a través de las mejoras o las novedades en la estructura dentro de la cual tienen lugar las actividades y procesos de la empresa, mientras que las segundas establecen nuevas relaciones con otros agentes, como las alianzas estratégicas, nuevos tipos de interfaz, etc.

En base a esta clasificación, la innovación en servicios se puede definir como la conversión de ideas en productos, procesos o servicios que el mercado valora. Estas ideas pueden ser tecnológicas, comerciales y organizacionales. Esta definición debe ser entendida en su sentido amplio, porque cubre todas las actividades que conllevan un cambio sustancial en la forma de hacer las cosas, incluyendo los servicios y productos que la empresa ofrece, así como la forma de producirlos, comercializarlos, distribuirlos y organizarlos (Jacob et al., 2003). Por tanto, las innovaciones tecnológicas son servicios nuevos o mejorados debido a la tecnología mientras que las innovaciones no tecnológicas consisten en nuevas formas de organización y gestión de las empresas o nuevos comportamientos en el mercado.

Aunque se ha insistido en la subestimación de las inversiones en I+D en el sector servicios parece generalmente aceptado que el sector servicios es menos innovador que el sector industrial. Sin embargo, no es del todo adecuado generalizar al referirnos al sector servicios en términos agregados cuando, en verdad, es un sector heterogéneo. Desde el punto de vista del esfuerzo innovador, Ducatel (2000) distingue entre tres tipos de servicios:

A.- Servicios dependientes de las redes de información, como son los financieros, los seguros, etc.

B.- Servicios empresariales intensivos en conocimiento, denominados KIBS en la literatura de innovación (Knowledge Intensive Business Services). Son muy similares a las empresas manufactureras en relación a la $\mathrm{I}+\mathrm{D}$ y la intensidad tecnológica. En este apartado destacan los servicios de software, diseño, consultoría avanzada, ingeniería, etc. 
C.- Servicios no intensivos en conocimiento e información, entre los que se encontrarían el comercio minorista, distribución, servicios de limpieza, turismo y otros.

En general, se sostiene, como hemos dicho anteriormente, la tradicional exclusión del mundo de la innovación de este tercer tipo de servicios entre los que se encuentra el turismo. En cierto sentido, se repite el argumento clásico desarrollado inicialmente para el sector manufacturero en el sentido de que las innovaciones se producían en este sector. Recientemente esta visión empieza a cambiar tanto en los servicios del tercer tipo en general como en el del turismo en particular. La inexistencia de trabajos aplicados al sector turismo no permitía refutar esta visión tradicional. A su vez, dada la contribución económica del turismo al empleo y a la producción en la mayoría de países de la OCDE, la falta de estudios de innovación en este sector no estaría justificada. Por ello, y dada la importancia del sector turístico balear, paradigma de destino turístico de masas de gran éxito, en los últimos cuatro años se ha hecho un esfuerzo de análisis de hasta qué punto su sector turístico en sus diferentes actividades desarrolla innovaciones (Jacob y Bravo, 2001; Jacob et al., 2003, 2004; Jacob y Groizard, 2007; Aguiló et al., 2003; Orfila-Sintes, 2003).

De ahí que en los próximos apartados nos centremos en los resultados de dos trabajos: por un lado, un estudio realizado el año 2003 sobre una muestra representativa de 300 empresas turísticas de Baleares (Jacob et al., 2004). Lógicamente, el trabajo pretendía dos cosas. En primer lugar, la valoración del tipo y característica de las innovaciones y su relevancia en dicho sector y, en segundo lugar, los motivos y las dificultades a las que se enfrentan las empresas para innovar. Por otro lado, un segundo estudio realizado en 2004 sobre una muestra de establecimientos hoteleros operados por empresas de origen balear en países de Latino-América y Caribe (LAC).

\section{METODOLOGÍA PARA LA CLASIFICACIÓN DE LAS EMPRESAS TURÍSTICAS Y SUS INNOVACIONES.}

En el primer estudio (Jacob et al., 2004) se utilizaron varios criterios para clasificar los tipos de empresas encuestadas, atendiendo a diferentes variables, En concreto, las empresas se clasificaron atendiendo a distintas características como son el año de fundación, el tipo de propiedad (independiente o perteneciente a un grupo empresarial), la localización de la sede del grupo empresarial, el tamaño de la empresa medido por el número de empleados, la cifra de facturación, el sector de actividad turística y el ámbito de operaciones (internacional, nacional, balear o insular).

El criterio de clasificación de las empresas según sector de actividad turística se basó en la concepción tradicional de la actividad turística como un compendio de diferentes actividades que pueden ser agrupadas atendiendo a la siguiente clasificación ${ }^{4}$ :

I Agencias de viajes: se incluyen las agencias de viaje minoristas y mayoristas.

\footnotetext{
4 Dentro del sector de transporte distinguimos entre empresas dedicadas al alquiler de vehículos y empresas de transporte propiamente dichas (empresas de autocares turísticos, empresas de charters aéreos, etc.) que presentan características muy distintas.
} 
I Hostelería: aparecen las empresas dedicadas al alojamiento, como hoteles, agroturismos, aparta-hoteles, etc.

I Restauración: aparecen las empresas dedicadas a la restauración como restaurantes y cafeterías.

I Ocio y Entretenimiento: están las empresas que se dedican a facilitar actividades de ocio a los turistas, como campos de golf, puertos deportivos, parques acuáticos, discotecas, etcétera.

I Transporte: Se incluyen las empresas de transporte discrecional orientadas al turismo y las compañías aéreas.

I Alquiler de vehículos: Se incluyen las empresas de alquiler de coches y de motocicletas.

A la hora de analizar las innovaciones introducidas por las empresas seleccionadas en las muestras de los dos trabajos mencionados (Jacob et al., 2004; Jacob y Groizard, 2007) también se emplean algunas variables de clasificación como son la innovación como objeto, la innovación como actividad y dentro de las innovaciones tecnológicas, éstas se clasifican adicionalmente atendiendo al área tecnológica de la innovación.

\section{Innovación como objeto:}

I Producto

I Proceso

I Comercialización

I Organización

I Mercado

\section{Innovación como actividad:}

I Tecnológica

I No tecnológica

Hay que recordar que estas innovaciones tecnológicas se clasifican adicionalmente según el área tecnológica en que se produzcan según la clasificación siguiente:

\section{Innovación tecnológica según área tecnológica:}

I Tecnologías de la Información y la Comunicación

I Sistemas de seguridad

I Medio Ambiente

I Cocinas

I Comedores y bares

I Sistemas de limpieza y lavandería

I Sistemas de jardinería 


\section{Salud y nutrición}

I Automatización del área de recepción

I Otras áreas tecnológicas

Además atendiendo al grado de novedad de las innovaciones, se tuvo en cuenta la clasificación tradicional de innovaciones radicales o incrementales. Las primeras incluyen la necesidad de que la innovación sea una auténtica novedad, mientras que las segundas solo implican que se produzca una mejora significativa. A su vez, el estudio incorpora la valoración de la llamada "exigencia tecnológica" o "seriedad tecnológica" que es un parámetro que mide el esfuerzo tecnológico de la empresa con la innovación introducida. Parece evidente que se exige mayor conocimiento tecnológico cuando la innovación comporta un mayor valor añadido tecnológico, por ejemplo, cuando se precisan unos recursos humanos más cualificados. En otros casos puede no ser necesario un elevado conocimiento tecnológico.

Otro criterio es la "capacidad de inducción tecnológica" que mide el potencial que tiene la innovación para impulsar innovaciones tecnológicas en otros sectores. Es probable que las innovaciones en servicios fomenten la creatividad innovadora en otras empresas, sobre todo tecnológica.

Finalmente hay que referirse al "potencial innovador" que es un parámetro que mide la capacidad de aumentar la rentabilidad del propio negocio, bien sea por un aumento de los ingresos o por una reducción de los costes.

\section{ALGUNOS RESULTADOS}

De las 300 empresas de Baleares entrevistadas en el primer estudio casi la mitad correspondían al sector hotelero, cerca de un $20 \%$ al de restauración y el resto a ocio, transporte, alquiler de vehículos y agencias de viajes.

A.- La innovación como objeto, actividad y área de tecnología. De los cinco tipos de innovación antes señalados significar que el $62 \%$ son innovaciones de proceso. Comercialización e innovación de producto e incluso organización interna poseen cierta relevancia, mientras que las innovaciones de organización externa y de mercado son prácticamente inexistentes. Por tanto, preponderancia total de las innovaciones tecnológicas (80\%), frente a las no tecnológicas (Cuadro 1).

En relación al área tecnológica, destacar que la mitad son las del área de las Tecnologías de la Información y Comunicación y casi el 20\% son innovaciones medioambientales.

B.- Innovación por sectores y tamaño. El sector de la hostelería contaba en la muestra de 300 empresas, un 46,7\%, no obstante del total de las innovaciones el 63,1\% correspondieron a este sector. Significativos también los porcentajes de los sectores de ocio y entretenimiento y restauración con un $13,7 \%$ y un $12,2 \%$ de las innovaciones, en cualquier caso algo inferiores a su segmentación en la muestra (Cuadro 2). 
De acuerdo con el tamaño según nivel de empleo, en las empresas pequeñas, hasta 49 empleados, se encuentra la mayor proporción de innovaciones con un 46,9\%. En cambio las empresas con facturación superior a 1.000 millones cuentan con el 26,75\% de las innovaciones aunque en la muestra sólo representan el 17\%.

Cuadro 1. Tipos de innovación en el sector turístico

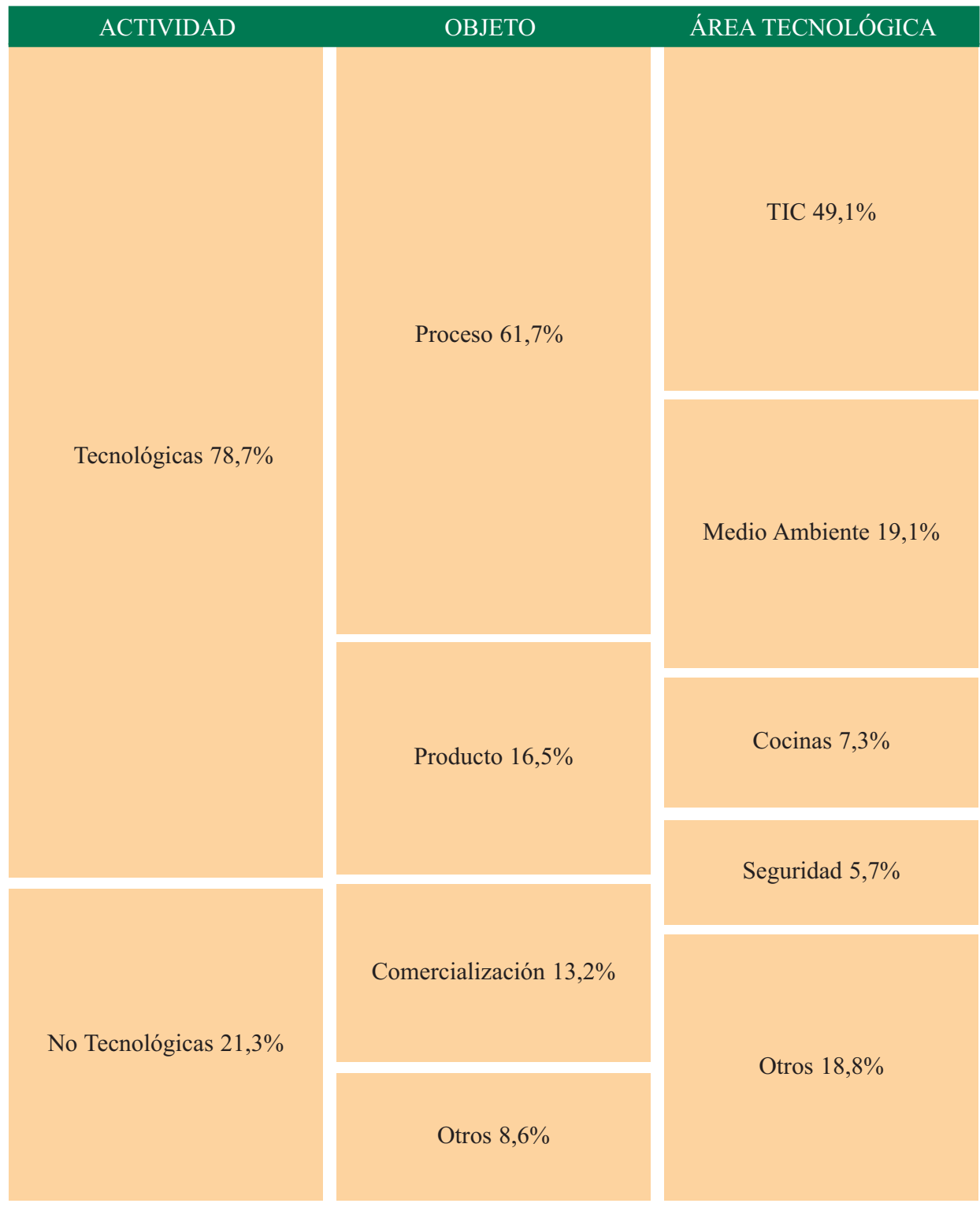


Cuadro 2. Importancia de la innovación por tipos de empresa

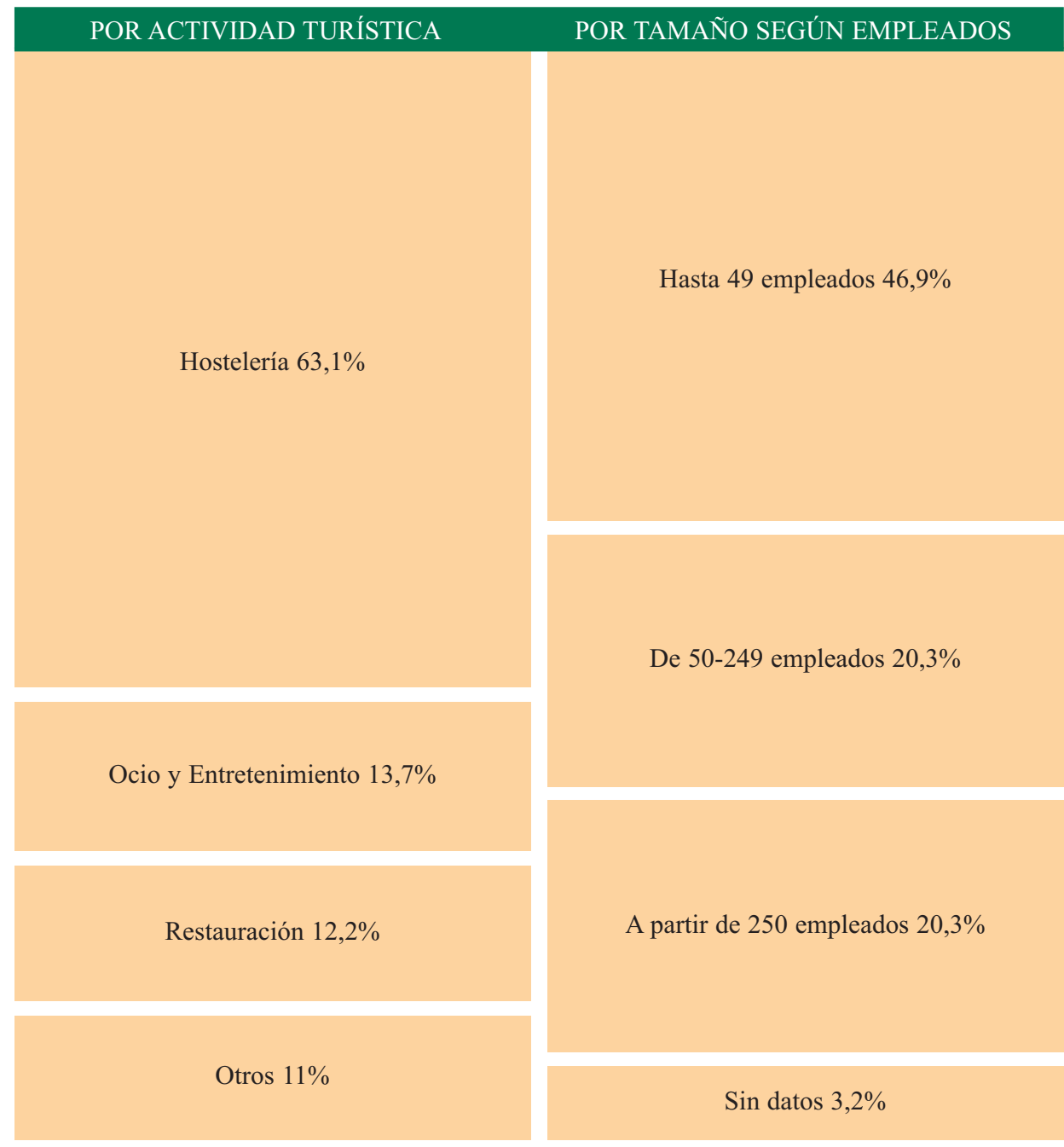

C.-Innovación por grado de novedad y potencial innovador. En el análisis del grado de novedad se observa que en todo el sector el $27,4 \%$ de las innovaciones introducidas (tecnológicas o no) presentan un grado de novedad elevado o muy elevado. En cambio el $30,1 \%$ y el $24,8 \%$ poseen un grado de novedad bajo o nulo, respectivamente. Por tanto, una de cada cuatro pueden calificarse de innovaciones radicales (Cuadro 3).

En cuanto al potencial innovador de las innovaciones introducidas en el sector turístico balear hay que señalar que casi un 50\% no producen aumento de ingresos y un $35,4 \%$ no suponen disminución de costes. En cambio, es cierto que una de cada cinco 
innovaciones presentan un potencial considerable de reducción de costes y también una de cada cinco, un potencial importante de generación de ingresos.

Cuadro 3. Innovación por grado de novedad y potencial innovador
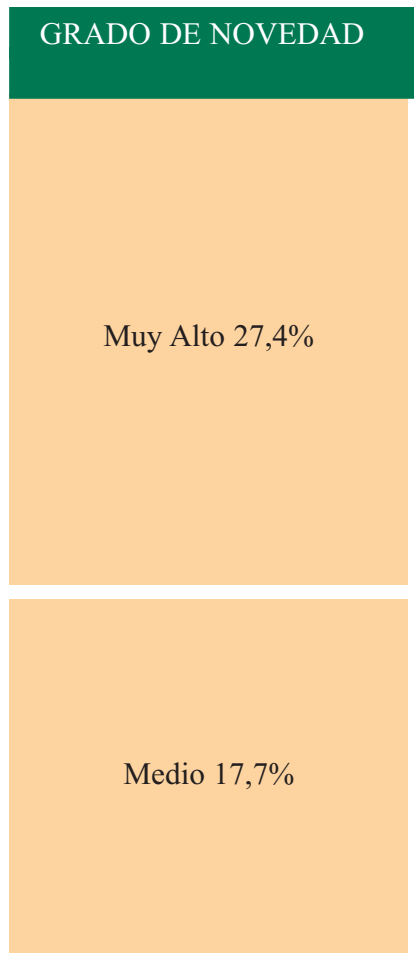

Bajo o nulo $78,9 \%$

Bajo o nulo $79,7 \%$

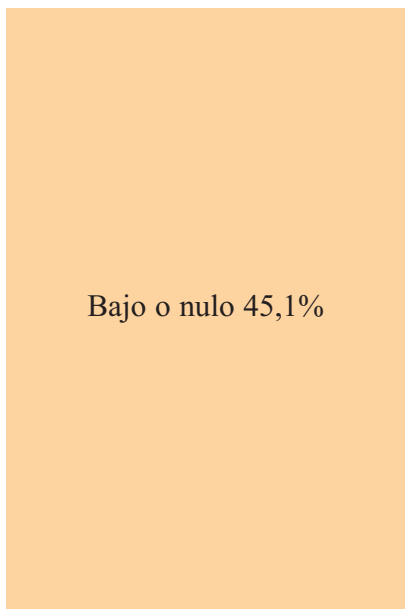

Medio 9,5\%

\section{POTENCIAL INNOVADOR}

Ingresos

Costes
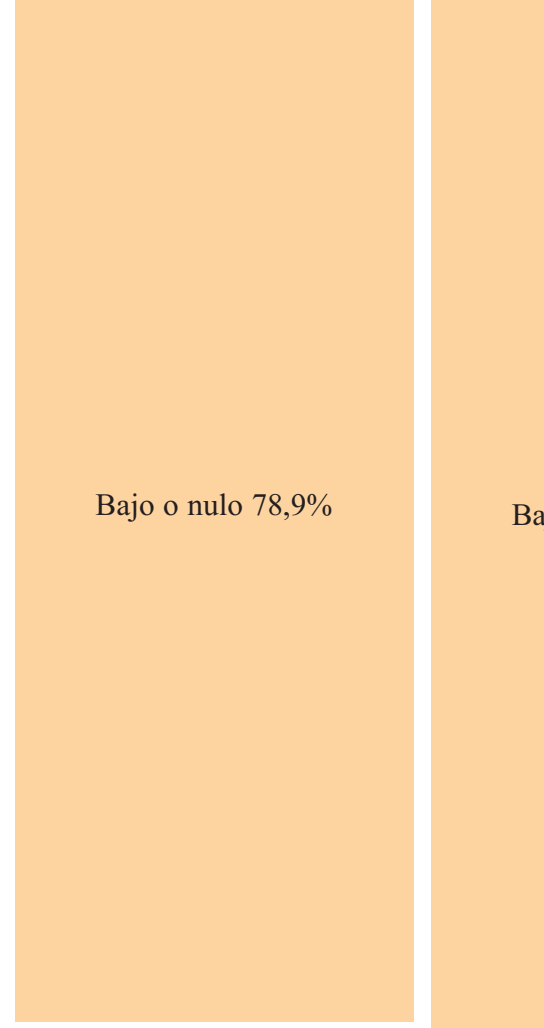
se observa que en el sector turístico balear en su conjunto sólo un porcentaje próximo al $15 \%$ de las innovaciones requiere un importante esfuerzo tecnológico por parte de las empresas Del resto, un 47,3\% requieren poco o incluso mínimo esfuerzo.

En cambio resulta interesante analizar la "capacidad de inducción tecnológica" en otros sectores económicos, ya que algo más del $40 \%$ muestran bastante o mucha capacidad de inducción, aunque en una de cada cuatro innovaciones esta capacidad es mínima

E.- Motivos, fuentes de información y otros factores de las actividades de innovación de las empresas.

En Baleares los principales motivos que inducen a la empresa a desarrollar una actividad innovadora son: satisfacer las necesidades del cliente y mejorar la calidad del servicio, lo cual está en consonancia con la actitud de las empresas del sector turístico de apostar claramente por la consecución de las certificaciones. Tampoco deben despreciarse los motivos de mejorar la imagen de la empresa, ser competitivo, mejorar la rentabilidad e incluso cumplir las regulaciones o normativas.

En el sector turístico balear hubo una coincidencia casi unánime en la dirección de la empresa como principal fuente de información de las innovaciones. Clientes y empleados también tuvieron su importancia, lo que demuestra que sus opiniones y sugerencias fueron tenidas en cuenta por las empresas. Conviene resaltar que las Universidades o Institutos de investigación son fuentes de información no relevantes para un porcentaje elevado de empresas.

Jacob et al. (2004) demuestran que las principales dificultades para innovar en las empresas turísticas de Baleares son la falta de personal (41\%) y el papel de la Administración pública (20\%) y el elevado coste $(20 \%)$.

¿Qué resultados esperan alcanzar las empresas al innovar? La evidencia empírica de Jacob et al. (2004) indica que la mejora de la satisfacción de los clientes y la mejora de la calidad del servicio son los principales resultados de la innovación. El mantenimiento o incremento de la cuota de mercado y la mejora de la productividad o la rentabilidad fueron dos razones citadas con cierta frecuencia aunque mucho menos que las dos anteriores.

Más del 60\% de las empresas turísticas manifestaban que la introducción de innovaciones ha sido positiva o muy positiva para la cifra de facturación. Algo menos del $50 \%$ han observado un impacto positivo sobre el empleo y cerca del 55\% detectaron una mejora del nivel de cualificación de sus trabajadores bien por una mayor y mejor formación de sus trabajadores o por la necesidad de contratar personal más formado o con cualificaciones especificas para poder desarrollar las innovaciones.

F.- Transferencia de tecnología de la industria hotelera balear a países menos desarrollados.

En Jacob y Groizard (2007) se realiza una comparación entre el número y tipo de tecnologías transferidas entre las empresas hoteleras de Baleares y sus establecimientos hoteleros localizados en dos destinos menos desarrollados en Latino-América y Caribe (México y República Dominicana) para documentar las diferencias en los patrones de 
innovación y absorción a nivel del establecimiento hotelero. La evidencia empírica se obtuvo a través de una encuesta on-line a los directivos de los hoteles de origen balear localizados en dos países de Latino-América y Caribe (LAC): México y Caribe, dicha evidencia se ha comparado con la obtenida en el estudio de Jacob et al. (2004). Los resultados indican que los hoteles pertenecientes a cadenas de origen balear y localizados en LAC son más innovadores que los localizados en Baleares, y esta diferencia es más significativa para los hoteles que operan desde 1990. Además, se encuentra una relación positiva entre la tasa de innovación del establecimiento y el tamaño del mismo, y cuando normalizamos el número de innovaciones por hotel por el número de habitaciones, los hoteles con más experiencia tienden a ser más innovadores. Los hoteles LAC introducen más innovaciones de proceso y organizacionales que los hoteles en Baleares pero este patrón no parece ser estable en los diferentes tamaños de hoteles. Finalmente, los hoteles en países LAC colaboran con empresas locales a la hora de introducir innovaciones y por ello, los establecimientos actualizan la cualificación de sus trabajadores con actividades de formación.

Todos estos resultados parecen confirmar que las actividades de innovación de las cadenas hoteleras multinacionales (como las de origen balear) en países de renta media y baja tienen un enorme potencial para promocionar el desarrollo doméstico. Cuanto más intensiva es la innovación, cuanto más codificada es la transferencia de conocimiento a sus hoteles subsidiarias, cuanto mayor formación y movilidad de los trabajadores y/o cuanto mayor colaboración con las empresas domésticas a la hora de crear o introducir innovaciones, mayor será la transferencia de conocimientos a las empresas domésticas. Los gobiernos locales pueden facilitar los beneficios en términos de incrementar la capacidad de un país de absorber las tecnologías en lugar de hacer que las cadenas hoteleras extranjeras inviertan en educación y, fortalecer los vínculos entre los agentes locales y extranjeros.

\section{CONCLUSIONES}

Este trabajo se ha iniciado con una serie de consideraciones en torno a la más reciente literatura sobre lo que representa la innovación en servicios.

La imposibilidad de generalizar en un mundo tan diverso como el de los servicios junto con lo novedoso de su tratamiento son las conclusiones básicas. A continuación se presenta la evidencia empírica disponible de dos estudios: un primer estudio que se centra en el análisis de la actividad innovadora de una muestra representativa de empresas turísticas de las Islas Baleares y un segundo estudio que analiza la transferencia de tecnología de otra muestra representativa de cadenas hoteleras de origen balear hacia países en vías de desarrollo donde tienen inversiones. Los resultados obtenidos del análisis del primer estudio (Jacob et al, 2004) indican varias pautas en la actividad innovadora del sector turístico balear. Básicamente, la innovación es un fenómeno extendido entre las empresas turísticas de Baleares, especialmente en el sector de hostelería. La tendencia a innovar aumenta con el tamaño, con la cifra de facturación o con el ámbito de operaciones de dichas empresas. La mayor parte de las innovaciones se dan en el proceso de producción y/o en la distribución y entrega del servicio. Aunque la mayoría de las inno- 
vaciones pueden ser consideradas como incrementales; sin embargo, una de cada cuatro son innovaciones radicales. Una de cada cinco de las innovaciones introducidas presentan un potencial considerable de reducción de costes y una de cada cinco muestra también un importante potencial de generación de ingresos. La innovación tecnológica o dependiente de tecnología es más frecuente que la no tecnológica, aunque una de cada cinco innovaciones introducidas es no tecnológica. Las innovaciones tecnológicas se concentran especialmente en el área de las tecnologías de la información y la comunicación, medio ambiente o salud y nutrición. Cerca del 15\% de las innovaciones tecnológicas introducidas requieren un importante esfuerzo tecnológico por parte de las empresas. Una proporción importante de las innovaciones introducidas en el conjunto del sector turístico balear presentan una capacidad considerable para inducir innovaciones en otros sectores económicos. La innovación medioambiental es un factor clave de competitividad de la industria turística en Baleares. Son frecuentes los desarrollos propios o con otros del equipamiento tecnológico necesario para introducir un cambio en la empresa. Los principales motivos para innovar son la satisfacción de las necesidades de los clientes y la mejora de la calidad del servicio. Las dificultades más importantes a la hora de innovar son la falta de personal cualificado y la actuación pública. Los principales efectos de la introducción de innovaciones son la mejora de la satisfacción del cliente y la mejora de la imagen y de la calidad del servicio ofertado.

Por último, los resultados del segundo estudio (Jacob y Groizard, 2007) sugiere que en el sector turístico las actividades de innovación de las cadenas hoteleras mutinacionales (como son las de origen balear) en países de renta media y baja tienen un enorme potencial para promocionar el desarrollo doméstico. La economía local receptora de estos países menos desarrollados se beneficia de las empresas multinacionales a través de la transferencia de conocimientos y de tecnologías. Los gobiernos locales pueden facilitar esa transferencia de tecnología y conocimientos.

\section{BIBLIOGRAFÍA}

Aguiló, E., Jacob, M., Mulet, J., Tintoré, J. \& Tortosa, E. (2003): “La innovación medioambiental como un factor básico de competitividad de las empresas turísticas de Baleares”, en Situación Islas Baleares. Serie Estudios Regionales 2003. Servicio Estudios BBVA, Madrid., pp. 311-328.

Aguiló, E., Alegre, J., \& Sard, M. (2005): “The persistence of the sun and sand tourism model". Tourism Management, 26(2): 219-231.

Ducatel, K. (2000): "Information Technologies in Non-Knowledge Services: Innovations on the Margin?" en Innovation Systems in the Service Economy; Measurement and Case Study Analysis; Economics of Science, Technology and Innovation, vol. 18, J.S. Metcalfe and I, Miles (editores), Kluwer Academic Publisher, USA, pp. 221-245.

EUROSTAT (1995): Report of the Eurostat Pilot Project to Investigate the Possibilities to Measure Innovation in the Service Sectors, Luxemburgo. 
Evangelista, R. \& Sirilli, G. (1995): "Measuring innovation in services", Research Evaluation, 5 (3): 207-215.

Gallouj, F. (1998): Innovation in Reverse Services and the Reverse Product Cycle, SI4S Topical Paper $n^{\circ}$ 5, STEP Group.

Hipp, C., Tether, B.S. \& Miles, I. (2000): "The Incidence and Effects of Innovation in Services: Evidence from Germany", International Journal of Innovation Management, 4 (4): 417-453.

Jacob, M. \& A. Bravo (2001): Estudio exploratorio sobre innovación en el sector turístico balear. Colección Estudios $n^{\circ} 21$. Fundación Cotec, Madrid.

Jacob, M. \& Groizard, J.L. (2007): “Technology transfer and multinationals: The case of Balearic hotel chains' investments in two developing economies", Tourism Management ,28: 976-992.

Jacob, M., Tintoré, J., Aguiló, E., Bravo, A. \& Mulet, J. (2003): “Innovation in the tourist sector: Results from a pilot study in the Balearic Islands". Tourism Economics, 9 (3): 279-295.

Jacob, M., Tintoré, J., Simonet, R. \& Aguiló, E. (2004): Pautas de innovación en el sector turístico balear. Colección Estudios n ${ }^{\circ} 25$, Fundación Cotec para la Innovación Tecnológica, Madrid.

Miles, I. (1994): "Innovation in Services. Part 2: Sectoral and Industrial Studies of Innovation” en: M. Dodgson y R. Rothwell (editors), The Handbook of Industrial Innovation, Edward Elgar, Reino Unido, pp 243-256.

Orfila-Sintes, F. (2003): La gestión del cambio tecnológico en la actividad hotelera. $\mathrm{PhD}$ Thesis, Universitat de les Illes Balears.

Sirilli, G. \& Evangelista, R. (1998): "Technological innovation in services and manufacturing: results form Italian surveys", Research Policy, 27 (9): 881-899.

Sundbo, J. \& Gallouj, F. (1998): Innovation in Services, SI14S Project Synthesis, WP 3-4.

Torres, X. \& Jacob, M. (2001): "La Innovación en Servicios" en Innovación Tecnológica. Ideas Básicas (E. Revilla Gutiérrez). Colección Innovación Práctica, Fundación Cotec, Madrid. 